International Journal of Bifurcation and Chaos, Vol. 21, No. 12 (2011) 3557-3564

(c) World Scientific Publishing Company

DOI: $10.1142 / \mathrm{S} 0218127411030726$

\title{
A DYNAMICAL APPROACH TO STOCK MARKET FLUCTUATIONS
}

\author{
S. C. NICOLIS* and D. J. T. SUMPTER ${ }^{\dagger}$ \\ Mathematics Department, \\ Uppsala University, Sweden \\ *snicolis@math.uu.se \\ †david@math.uu.se
}

Received September 5, 2010; Revised January 21, 2011

\begin{abstract}
The recent turbulence on the world's stock markets has reinvigorated the attack on classical economic models of stock market fluctuations. The key problem is determining a dynamic model, which is consistent with observed fluctuations and which reflects investor behavior. Here, we use a novel equation-free approach developed in nonlinear dynamics literature to identify the salient statistical features of fluctuations of the Dow Jones Industrial Average over the past 80 years. We then develop a minimal dynamical model in the form of a stochastic differential equation involving both additive and multiplicative system-noise couplings, which captures these features and whose parameterization on a time scale of days can be used to capture market distributions up to a time scale of months. The terms in the model can be directly linked to "herding" behavior on the part of traders. However, we show that parameters in this model have changed over a number of decades producing different market regimes. This result partially explains how, during some periods of history, "classic" economic models may work well and at other periods "econo-physics" models prove better.
\end{abstract}

Keywords: Econophysics; stochastic processes; extreme events.

\section{Introduction}

Financial activities as examplified by stock market fluctuations constitute a highly relevant example of complex nonlinear behavior in human systems. They highlight the intertwining, on a global scale, of order and disorder despite the supposedly rationality-driven motivations and actions of the actors at the individual level. Clearly, monitoring and prediction become here key issues.

The classical view of stock market growth postulates that market returns are independent of each other over anything but the very shortest of time scales [Samuelson, 1965; Fama, 1965]. This postulation leads one to map stock market growth to
Brownian motion, one of the most familiar problems in the theory of stochastic processes. In practice, to account for the undeniable variation in the size of fluctuations on stock markets, the variance of these models is stipulated to change dynamically. Important families of models in this respect are ARCH, GARCH and stochastic volatility models [Engle, 1982, 1995; Chernov et al., 2003] which assume that the variance of the current market return is a function of the magnitude of recent previous returns. Put in terms of human behavior, these models reflect the observation that large changes in the market generate uncertainty leading to further uncertainty and more fluctuations.

*Present address 
During the last decades, stock market behavior has been revisited using ideas and tools from the theory of stochastic processes and statistical mechanics as applied to critical phenomena. In this view, the limitations of both the Brownian motion and GARCH style models are pointed out and emphasis is placed instead on the observation that some of the most characteristic indicators of financial activities display long-range correlations and follow fractal scaling laws over several orders of magnitude. Such properties are reminiscent of multifractal processes [Vandewalle \& Ausloos, 1998; Calvet \& Fisher, 2001] and have on these grounds been modeled as multiplicative cascade processes [Schmitt et al., 2000]. They are also compatible with a Levy stable distribution [Buchanan, 2009; Mandelbrot, 1963; Mantegna \& Stanley, 1995]. One important characteristic of the Levy-stable distribution is that it has a power law tail with exponent less than 3, thus predicting the very large fluctuations, crashes and rallies, of the kind seen in stock markets [Mandelbrot, 1966; Blanchard, 1979; Bouchaud \& Cont, 1998; Sornette \& Malevergne, 2001; Sornette \& Johansen, 1997].

In fact, the exponents in distributions of market fluctuations are usually more than 3, and GARCH models also produce power law tails consistent with data for a given time scale [Sornette, 2001; Dehaan et al., 1989]. However, GARCH models have a fixed time scale and are usually not consistent with the data on a range of time scales [Farmer, 1999]. We are thus left with two types of view: Levy laws and multifractal models that potentially capture scaling behavior of returns and related quantities; and GARCH models which give an ad-hoc means of modeling fluctuations on a given time scale. Furthermore, neither of these models are explainable in terms of trader behavior. The question of providing a dynamic model that reflects the mechanisms at work in stock trading and reproduces market returns over multiple time scales remains open [Farmer, 1999; Stanley et al., 2002; Farmer \& Foley, 2009].

In this paper stock market is viewed in a dynamical perspective. We derive a set of phenomenological evolution laws of key variables generating some salient features of the data in a self-consistent manner. The aim is to identify key mechanisms and to propose useful ways to characterize the system at hand. The predictive potential of the approach will also be assessed.

\section{Data Analysis}

We first adopt an "equation free" approach recently developed in the nonlinear dynamics literature to extract quantities relevant to market dynamics [Yates et al., 2009; Bindal et al., 2006; Erban et al., 2006; Haataja et al., 2004]. A first question in this context is how the mean and standard deviation of the returns on one day, week or month depend upon the returns on the previous day, week or month. A market return $x(t)$ is defined to be

$$
x(t)=\frac{y(t+1)-y(t)}{y(t)}
$$

where $y(t)$ and $y(t+1)$ are the actual market indexes at time $t$ and $t+1$.

The index we investigate is the Dow Jones Industrial Average for the entire period October 1st 1928 to September 11th 2009. A typical time series of the corresponding returns is depicted in Fig. 1. We start by simply plotting returns for consecutive time steps (daily, weekly or monthly) against each

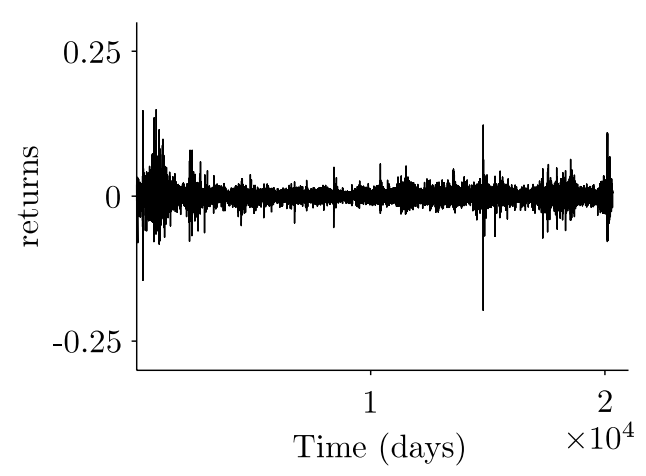

(a)

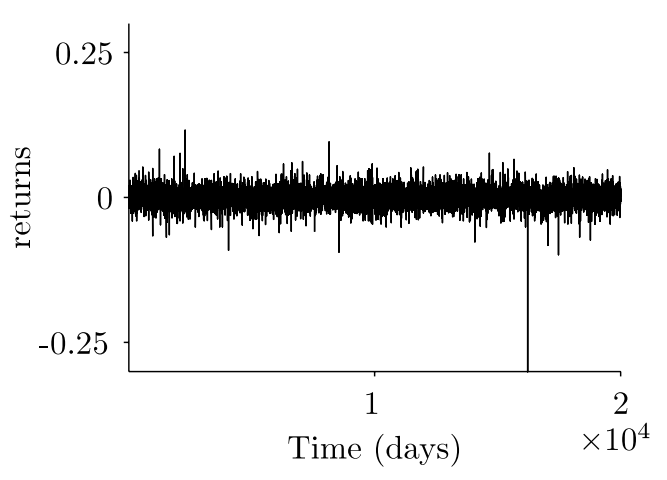

(b)

Fig. 1. Time series of daily return values of Dow Jones Industrial Average (a) from the entire period 1st October 1928 to 11th September 2009 and (b) time series generated by the model of Eq. (1). 
other, in order to infer the structure of

$$
f(x(t)) \equiv \mathrm{E}[x(t+1) \mid x(t)]
$$

[Figs. 2(a), 2(d) and 2(g)]. Furthermore, we plot variability over one time step

$$
s(x(t), x(t+1))=\sqrt{(x(t+1)-x(t)-f(x(t)))^{2}}
$$

[Figs. 2(b), 2(e) and 2(h)], along with the frequency distribution of return sizes [Figs. 2(c), 2(f) and 2(i)].

As can be seen, there are surprisingly clear patterns in both the mean and variance of changes in returns, as well as the distribution of returns: (1) For small daily returns, $f(x(t))$ depends in the mean linearly on $x(t)$, i.e. $f(x(t)) \approx \gamma x(t)$. This pattern is less clear in the case of larger returns

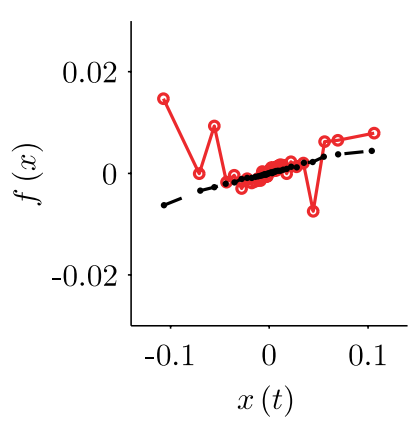

(a)

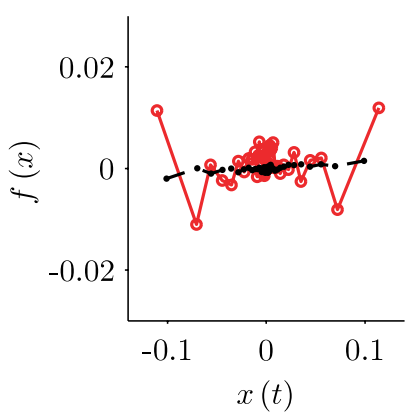

(d)

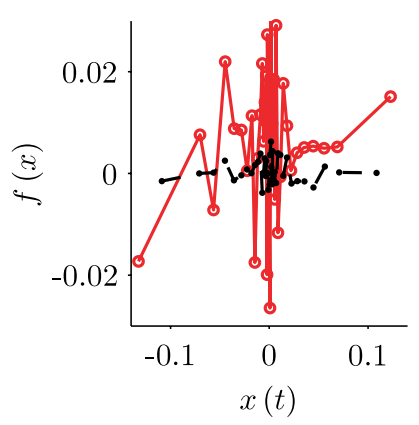

(g)

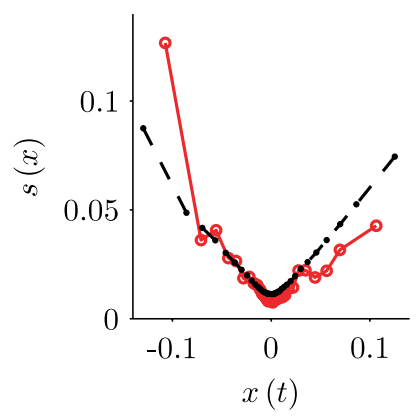

(b)

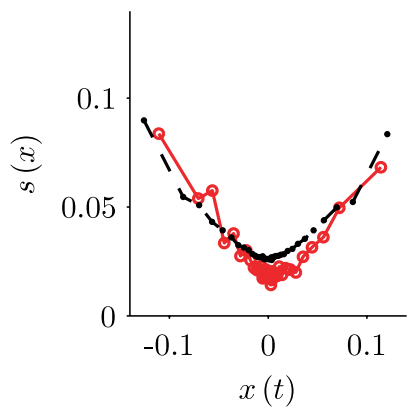

(e)

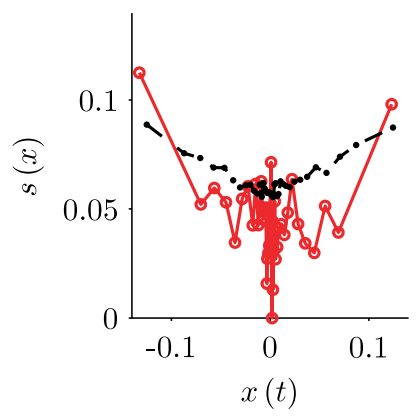

(h)

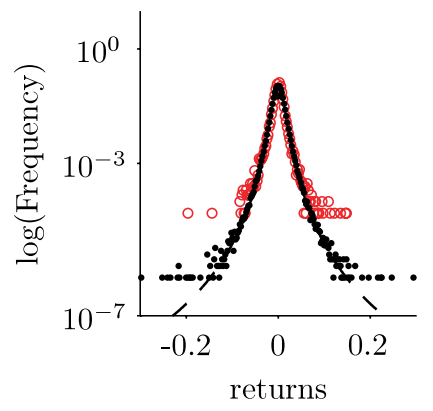

(c)

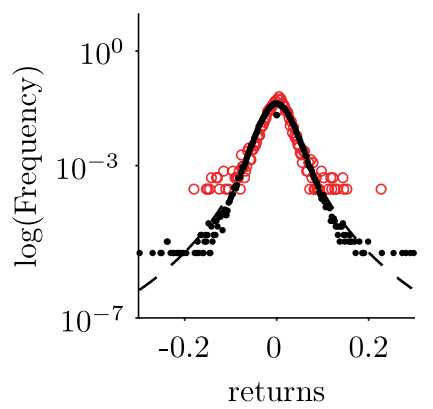

(f)

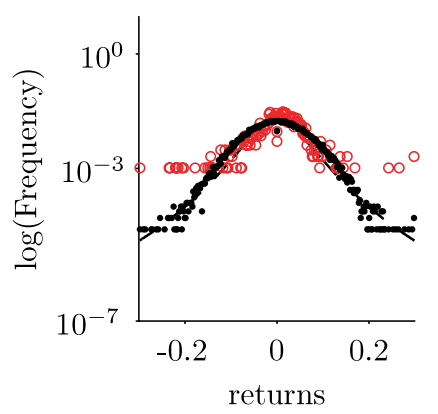

(i)

Fig. 2. $f(x)$ versus $x(t)$, standard deviation of the drift $s(x)$ versus $x(t)$ and probability distribution of daily (a, b, c), weekly $(\mathrm{d}, \mathrm{e}, \mathrm{f})$ and monthly $(\mathrm{g}, \mathrm{h}, \mathrm{i})$ returns. Data in red, model in black. Parameter values used on the basis of the daily data and equal to $\alpha=3.01, q_{1}^{2}=2.01 \times 10^{-1}$ and $q_{2}^{2}=5.29 \times 10^{-4}$. The predicted distribution of returns (dashed lines) are fitted with the actual values of the parameters: $\alpha=3.01, q_{1}^{2}=2.01 \times 10^{-1}$ and $q_{2}^{2}=5.29 \times 10^{-4}$ for daily returns; $\alpha=5.68$, $q_{1}^{2}=9.14 \times 10^{-2}$ and $q_{2}^{2}=5.39 \times 10^{-3}$ for weekly returns; and $\alpha=1.79, q_{1}^{2}=1.77 \times 10^{-1}$ and $q_{2}^{2}=1.20 \times 10^{-2}$ for monthly returns. Owing to highly inhomogeneous sampling of the data, the convention adopted for the binning is for negative values where $d$ varies from 1 to 2.8 by steps of 0.1 and symmetrically for positive $x$ 's. 
and disappears altogether for weekly and monthly returns; (2) the standard deviation displays a V-like dependence as a function of $x(t)$, each branch of which increases in a roughly linear fashion with the size of the previous return; (3) the distribution for daily returns is sharply peaked but with "fat tails", while for months the distribution is wide but bell-shaped.

Preliminary information on the nature of variability displayed by a time series is also provided by the time autocorrelation function. As it turns out, autocorrelation of the time series of the returns per se [Fig. 1(a)] drops quite rapidly to zero. The situation is different for the time autocorrelation of the absolute values of the returns, a quantity related to market volatility. After a fast initial drop, this quantity persists at non-negligible values over a long period of time. This is in agreement with results previously reported in the econophysics literature [Arnéodo et al., 1998; Grau-Carles, 2001; Podobnik et al., 2009].

\section{Model}

A minimal stochastic dynamical model which accounts for some salient features as summarized above is as follows. We assume a linear (in the mean) relationship between returns on consecutive days. Intrinsic variability in this relationship is captured by modeling the fluctuations in the slope of the linear law as a Markov noise process $F_{1}(t)$. Extrinsic variability generated by the environment is then modeled by an additive Markov noise process $F_{2}(t)$. We thus arrive at the following continuous-time stochastic differential equation governing the evolution of $x$,

$$
\frac{d x}{d t}=-\alpha\left(1+F_{1}(t)\right) x+F_{2}(t)
$$

where $F_{1}(t)$ and $F_{2}(t)$ will from now on be assimilated to Gaussian white noises with variances equal to $q_{1}^{2}$ and $q_{2}^{2}$, respectively.

As well known, a stochastic differential equation driven by white noise can be interpreted in the sense of Stratonovitch or in the sense of Itô depending on whether the white noise is viewed as the limit of colored noise or the continuous time formulation is viewed as the limit of a discrete time one. In most physical applications, the Stratonovitch turns out to be the appropriate one [van Kampen, 1981]. Nevertheless, we shall here adopt the Itô interpretation as widely used in the finance literature on the grounds of causality and therefore of the no-arbitrage condition. The probability distibution of $x$ obeys then a Fokker-Planck equation of the form [van Kampen, 1981]

$$
\frac{\partial P}{\partial t}=\frac{\partial}{\partial x} \alpha x P+\frac{1}{2} \frac{\partial^{2}}{\partial x^{2}}\left(q_{1}^{2} \alpha^{2} x^{2}+q_{2}^{2}\right) P
$$

where the first term is the drift and the second one is the (generalized) diffusion. The first, second and fourth moment equations can be deduced from Eq. (2)

$$
\begin{aligned}
& \frac{d \bar{x}}{d t}=-\alpha \bar{x} \\
& \frac{d \overline{x^{2}}}{d t}=-2 \alpha\left(1-\frac{\alpha q_{1}^{2}}{2}\right) \overline{x^{2}}+q_{2}^{2} \\
& \frac{d \overline{x^{4}}}{d t}=-4 \alpha\left(1-\frac{3}{2} \alpha q_{1}^{2}\right) \overline{x^{4}}+6 q_{2}^{2} \overline{x^{2}} .
\end{aligned}
$$

According to Eqs. (3a) and (3b), the mean value is always stable as long as $\alpha>0$ and the fluctuations become unstable if $\alpha q_{1}^{2}>2$. Finally, Eq. (2) admits the following steady state solution

$$
P \approx\left[1+\frac{\alpha^{2} q_{1}^{2}}{q_{2}^{2}} x^{2}\right]^{-\left(1+1 / \alpha q_{1}^{2}\right)} .
$$

Equation (4) belongs to the class of $q$-Gaussians which have recently been used extensively in the complex systems literature [Mendes \& Tsallis, 2001]. It can, depending on the parameter values, take both a power law form and a narrow distribution. In particular, one recovers a power law type of relation if $\left(q_{1}^{2} \alpha^{2} / q_{2}^{2}\right) x^{2} \gg 1$. The existence of finite first few moments of (4) requires that $q_{1}^{2} \alpha$ be sufficiently small, corresponding to the condition of moment stability in Eqs. (3b) and (3c). Note that the variables in Eq. (4) can be rescaled to give Student's t distribution. Student's t is widely used for modeling financial data precisely because of its versatility with respect to its tails [Papoulis, 1990]. Our derivation provides a means of parameterizing this model in terms of return dynamics. We stress that adding nonlinearities in the drift term in Eqs. (1) and (2) would not lead to a qualitatively new situation as long as no "phase transition" occurs, i.e. as long as the first moment equation (3a) admits $\bar{x}=0$ as the sole steady state. There is no evidence from the data that this property may not be satisfied in the present problem. 


\section{Results}

We now summarize the main predictions of the model and compare them with the data. The first step is to evaluate the three model parameters $\alpha, q_{1}$ and $q_{2}$. We do this by setting the moment Eqs. (3b) and $(3 \mathrm{c})$ equal to zero and solving the parameters in terms of the moments of the returns data. We then use the first moment equation to obtain by straightforward integration the slope $\gamma$ of $x(t+1)$ versus $x(t)$ as $\gamma=\exp (-\alpha)$. Using this information we obtain an explicit form of the probability of the process [Eq. (4)] and also carry out a stochastic simulation of Eq. (1) from which the remaining quantities displayed in Fig. 2 are inferred. Notice that the parameters are determined from daily data and then compared to weekly and monthly data, showing that the model scales well between these time scales.

A typical time series generated by the model over a time period of the same length as the original record is shown in Fig. 1(b). Compared to Fig. 1(a) it looks more uniform and more stationary. It still gives rise, however, to bursts reminiscent of stock market data, including a marked crash (right part of the record). Further results of the model are indicated as black dashed lines in Fig. 2. The model accurately reproduces a large number of features seen in the data: (1) correlations in returns seen over days disappear over weeks and months; (2) correlations in the variance of the returns weaken but remain visible as the time scale is lengthened; and (3) the return distribution transforms from having a steep point and long tail for days to a wide distribution for months.

We turn next to the time autocorrelation functions of the returns and of their absolute values. The first of these quantities shows quite similar behavior to that drawn from the data. In contrast, the second quantity drops rapidly to zero. This absence of persistence as compared to the data is due to the fact that the model of Eq. (1) is driven by Gaussian white noises and gives thus rise to a continuous-time Markov (diffusion) process, even though one of the noises acts in a multiplicative fashion. To reproduce long tail behavior of volatility correlations while keeping return correlations short ranged, additional features would be needed, which are beyond the scope of the present model. Actually, most of the currently available models reproducing long-range behavior of volatilities are not reducible to a dynamical systems formulation as in our Eq. (1) but are either ad hoc multiplicative cascades models [Arnéodo et al., 1998] or game theory based models [Bouchaud et al., 2001].

All in all, the model gives a good match of salient features of the data over 80 years. On the other hand, it would be surprising if the parameters remain constant over this time. In Fig. 3, we plot year by year parameter estimates for our model. It is interesting to see, for example, that the historically documented instability of the Dow Jones between 1929 and 1932 can be characterized by a relatively high $q_{2}^{2} / \alpha$, possibly reflecting the enhancement of environmental noise. The values of $q_{1}^{2} \alpha$ are relatively high for the crash of 1987 and the economic crisis of 2008, reflecting increased correlations between consecutive returns. As $q_{1}^{2} \alpha$ approaches the value of 2 the second moment of the return distribution ceases to exist and large deviations in market returns become possible. For 1987, our model gives a distribution with a tail characterized by a power law with exponent of 5.31 .

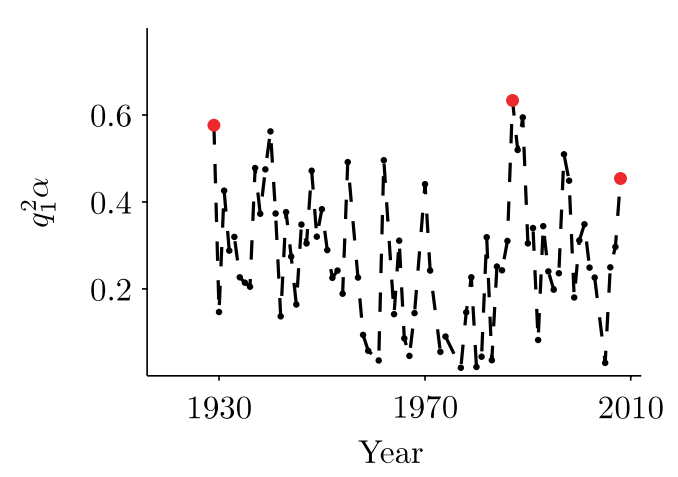

(a)

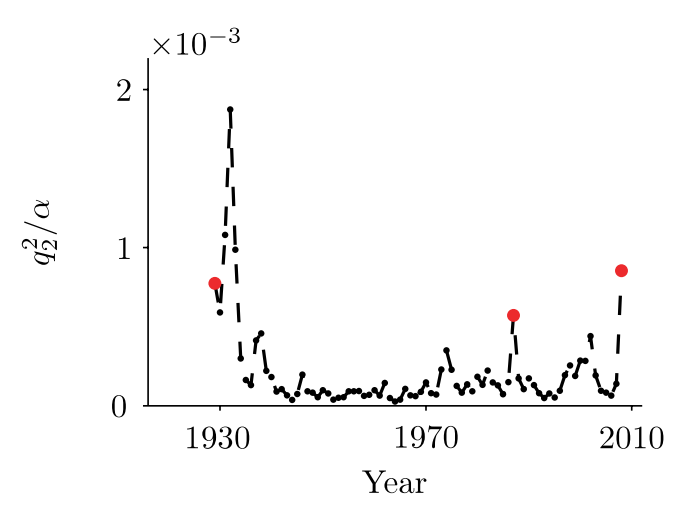

(b)

Fig. 3. Evolution of the parameters of the model year by year. Gray dots denote important historical crash. Years where $q_{1}^{2}<0$ were removed and years where the data slope was negative was taken in absolute values. 
This observation explains, in a dynamic context, the near Levy-walk like behavior of the financial markets during the mid-80s [Mantegna \& Stanley, 1995]. While many previous studies have emphasized these power law tails in stock return distribution, one can see that there are extensive periods of time where the prefactor in Eq. (4), $q_{1}^{2} \alpha^{2} / q_{2}^{2}$, is relatively small and power law like behavior is recovered only for extreme values of $x$. Our model thus provides a characterization of the entire dynamics of returns in terms of the intrinsic parameters built in a dynamical model and has in this respect a predictive potential.

Another important question is how the functional form of $f(x)$ changes over time. Figure 4 summarizes the behavior of the quantities considered in Fig. 2 for more limited periods spanning the 60s, 80 s and 2000s. For the 60s, the linear dependence in $f(x)$ is particularly clear. For the 80 s, the slope $\gamma$ is near to zero for small returns but becomes visible again for returns larger than $1 \%$. During the 2000s, we see a negative slope $\gamma<0$. In this latter

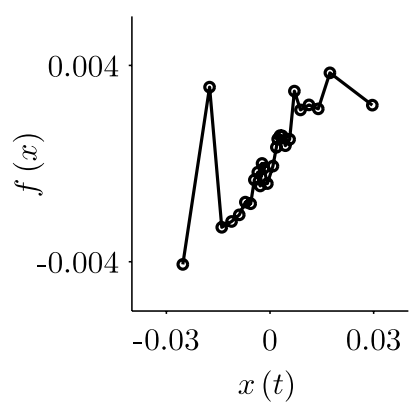

(a)

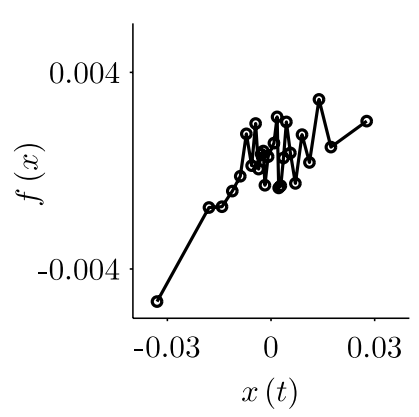

(d)

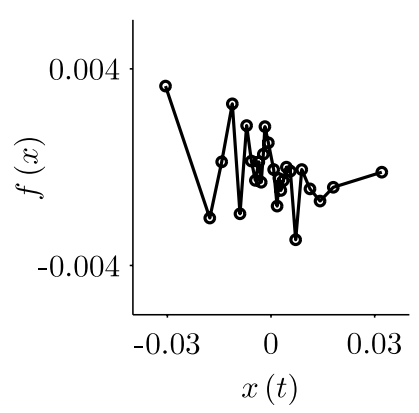

(g)

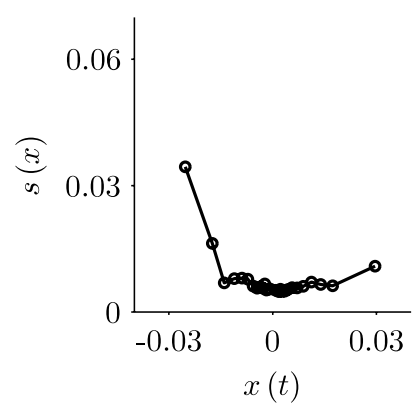

(b)

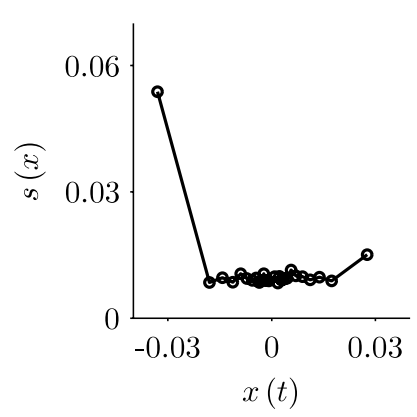

(e)

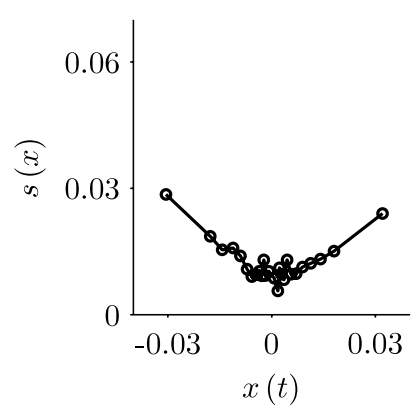

(h)

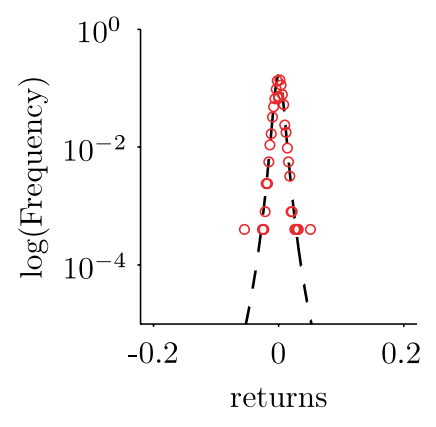

(c)

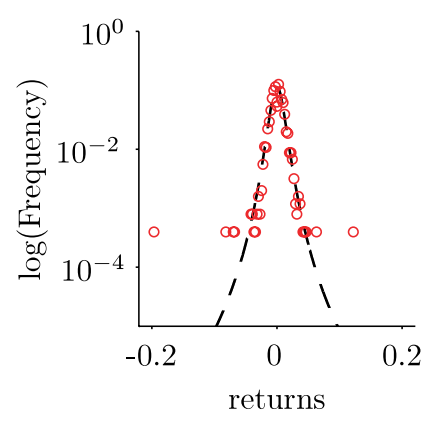

(f)

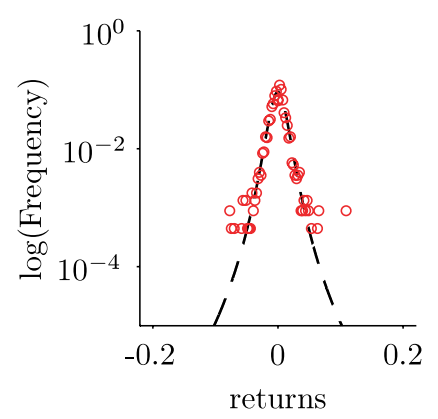

(i)

Fig. 4. $f(x)$ versus $x(t)$, standard deviation of the drift $s(x)$ versus $x(t)$ and probability distribution of daily returns for the 60's (a, b, c), 80's (d, e, f) and 2000's (g, h, i). The theoretical probability distribution is plotted in dashed line with parameter values taken from the data: $\alpha=1.72, q_{1}^{2}=2.63 \times 10^{-1}$ and $q_{2}^{2}=1.09 \times 10^{-4}$ for the 60 's, $\alpha=3.25, q_{1}^{2}=1.97 \times 10^{-1}$ and $q_{2}^{2}=5.22 \times 10^{-4}$ for the 80's and $\alpha=2.66, q_{1}^{2}=2.05 \times 10^{-1}$ and $q_{2}^{2}=6.30 \times 10^{-4}$. Binning same as Fig. 1. 
case, Eq. (1) fails to predict the negative slope, although it does reproduce the overall return distribution. Basically, a negative slope implies nonmonotonic behavior in time, the modeling of which would require at least one additional dynamical variable.

It is important to put the model in the context of the wide range of models already proposed for stock market fluctuations. For example, an $\mathrm{ARCH}(1)$ model can be fit to the variance in Figs. 2(a), 2(d) and 2(g). Such a fitting does not however result in a good prediction of the weekly and monthly returns, because the time scale is omitted from this model. Our model can also be viewed as a continuous time version of a stochastic difference equation known as the Kesten process [Sornette \& Malevergne, 2001; Kesten, 1973; Sornette, 2004]. Furthermore, it shares common features with some of those proposed in econo-physics. An equation similar to (1) but with no additive noise term (i.e. $q_{2}^{2}=0$ ) was considered by Richmond [2001] as a possible origin of power law tails in financial time series, but he provided no clear empirical justification. Now, Eq. (4) gives rise to power law behavior only in a range of large deviations. Furthermore, the signature of financial crisis resides not only in the value of the exponent but also on the parameter $\alpha q_{1}^{2}$ controlling the stability of the moments. Indeed, reincorporating the additive noise and providing the relationship $\alpha q_{1}^{2}>2$, we provide a clear relationship between Eq. (1) and market crashes, in the sense that all moments but the first one are unstable.

\section{Conclusions}

In conclusion, one of the merits of the combined equation-free and model driven approaches developed in this work is to provide a unifying view of market dynamics reducing in appropriate limits to classical and to critical dynamics type of approaches, and to propose a decomposition of its salient features in terms of three key parameters, $\alpha, \alpha q_{1}^{2}$ and $q_{2}^{2} / \alpha$. Thanks to the equation-free approach, figures such as Fig. 2 highlight pertinent features of stock market returns [Bindal et al., 2006; Erban et al., 2006; Haataja et al., 2004]. Once recurrent patterns are found, characterization of the kind we used in Eqs. (1)-(4) and in Fig. 2 can be made via a model. Indeed, the features of the stochastic dynamical model we have derived allow us to better understand the behavior of the stock traders.
A positive slope of the deterministic term $\alpha$ indicates a degree of herding behavior with respect to daily returns, rather than the market value, acting as stimulus for further increases. Usually, this herding is subsumed by the intrinsic noise $\alpha q_{1}^{2}$, which reflects traders making large, correlated changes to their buying or selling behaviour in response to large market changes. Finally, $q_{2}^{2} / \alpha$ is external noise generated by genuine uncertainty about how changes in the world will affect the values of stock. In the present work both kinds of noise sources have been assimilated to Gaussian white noises. It would undoubtedly be interesting to extend the analysis to colored noises and to assess the role of correlation and memory effects in stock market dynamics.

Finally, we believe that the methodology developed in this paper is quite generic and can as such be applied to a variety of phenomena beyond the stock market fluctuations, where variability is a ubiquitous feature. Of special interest is the variability of atmospheric fields where, depending on the scale, long range correlations and power laws coexist with white noise processes and narrow distributions. In view of the uncertainties underlying the determination of the initial conditions and of the parameters needed to run traditional numerical prediction models, data analyses in the spirit of the equation-free approach used here should provide a valuable complement.

\section{Acknowledgment}

We gratefully acknowledge financial support from the Human Frontier Science Program.

\section{References}

Arnéodo, A., Muzy, J. F. \& Sornette, D. [1998] "Direct causal cascade in the stock market," Eur. Phys. J. B 2, 277-282.

Bindal, A., Ierapetritou, M., Balakrishnan, S., Armaou, A., Makeev, A. \& Kevrekidis, I. [2006] "Equation-free, coarse-grained computational optimization using timesteppers," Chem. Eng. Sci. 61, 779-793.

Blanchard, O. J. [1979] "Speculative bubbles, crashes and rational-expectations," Econom. Lett. 3, 387-389.

Bouchaud, J. P. \& Cont, R. [1998] "A Langevin approach to stock market fluctuations and crashes," Europ. Phys. J. B 6, 543-550.

Bouchaud, J. P., Giardina, I. \& Mézard, M. [2001] "On a universal mechanism for long-range volatility correlations," Quant. Finan. 1, 212-216. 
Buchanan, M. [2009] "Economics: Meltdown modelling," Nature 460, 680-682.

Calvet, L. \& Fisher, A. [2001] "Forecasting multifractal volatility," J. Econometr. 105, 27-58.

Chernov, M., Gallant, A. R., Ghysels, E. \& Tauchen, G. [2003] "Alternative models for stock price dynamics," J. Econometr. 116, 225-257.

Dehaan, L., Resnick, S. I., Rootzen, H. \& Devries, C. G. [1989] "Extremal behavior of solutions to a stochastic difference equation with applications to arch processes," Stochast. Process. Their Appl. 32, 213-224.

Engle, R. F. [1982] "Autoregressive conditional heteroscedasticity with estimates of the variance of united-kingdom inflation," Econometrica 50, 9871007.

Engle, R. F. [1995] ARCH: Selected Readings, Advanced Texts in Econometrics (Oxford University Press, Oxford, NY).

Erban, R., Kevrekidis, I., Adalsteinsson, D. \& Elston, T. [2006] "Gene regulatory networks: A coarse-grained, equation-free approach to multiscale computation," J. Chem. Phys. 124, 084106.

Fama, E. F. [1965] "The behavior of stock-market prices," J. Business 38, 34-105.

Farmer, J. D. [1999] "Physicists attempt to scale the ivory towers of finance," Comput. Sci. Eng. 1, 26-39.

Farmer, J. D. \& Foley, D. [2009] "The economy needs agent-based modelling," Nature 460, 685-686.

Grau-Carles, P. [2001] "Long-range power-law correlations in stock returns," Physica A: Statist. Mech. Appl. 299, 521-527.

Haataja, M., Srolovitz, D. \& Kevrekidis, I. [2004] "Apparent hysteresis in a driven system with selforganized drag," Phys. Rev. Lett. 92, 160603.

Kesten, H. [1973] "Random difference equations and renewal theory for products of random matrices," Acta Mathematica 131, 207-248.

Mandelbrot, B. [1963] "The variation of certain speculative prices," J. Business 36, 394-419.

Mandelbrot, B. [1966] "Forecasts of future prices, unbiased markets, and martingale models," J. Business 39, 242-255.
Mantegna, R. \& Stanley, H. E. [1995] "Scaling behavior in the dynamics of an economic index," Nature $\mathbf{3 7 6}$, 46-49.

Mendes, R. \& Tsallis, C. [2001] "Renormalization group approach to nonextensive statistical mechanics," Phys. Lett. A 285, 273-278.

Papoulis, A. [1990] Probability and Statistics (Prentice Hall, New Jersey).

Podobnik, B., Horvatic, D., Petersen, A. M. \& Stanley, H. E. [2009] "Cross-correlations between volume change and price change," Proc. Natl. Acad. Sci. 106, 22079-22084.

Richmond, P. [2001] "Power law distributions and dynamic behaviour of stock markets," Europ. Phys. J. B 20, 523-526.

Samuelson, P. A. [1965] "Proof that properly anticipated prices fluctuate randomly," Imr-Industrial Management Review 6, 41-49.

Schmitt, F., Schertzer, D. \& Lovejoy, S. [2000] "Multifractal fluctuations in finance," Int. J. Theoret. Appl. Finan. 3, 361-364.

Sornette, D. [2001] "Fokker-planck equation of distributions of financial returns and power laws," Physica A - Statist. Mech. Appl. 290, 211-217.

Sornette, D. [2004] Critical Phenomena in Natural Sciences, 2nd edition (Springer Verlag).

Sornette, D. \& Johansen, A. [1997] "Large financial crashes," Physica A 245, 411-422.

Sornette, D. \& Malevergne, Y. [2001] "From rational bubbles to crashes," Physica A 299, 40-59.

Stanley, H. E., Amaral, L. A. N., Buldyrev, S. V., Gopikrishnan, P., Plerou, V. \& Salinger, M. A. [2002] "Self-organized complexity in economics and finance," Proc. Nat. Acad. Sci. USA 99, 2561-2565.

van Kampen, N. G. [1981] Stochastic Processes in Physics and Chemistry (North-Holland).

Vandewalle, N. \& Ausloos, M. [1998] "Multi-affine analysis of typical currency exchange rates," Europ. Phys. J. B 4, 257-261.

Yates, C. A., Erban, R., Escudero, C., Couzin, I. D., Buhl, J., Kevrekidis, I. G., Maini, P. K. \& Sumpter, D. J. T. [2009] "Inherent noise can facilitate coherence in collective swarm motion," Proc. Nat. Acad. Sci. USA 106, 5464-5469. 